\title{
Molecular analysis of ovarian mucinous carcinoma reveals different cell of origins
}

\author{
Yihong Wang ${ }^{1,2}$, Lauren Ende Shwartz ${ }^{2}$, Derek Anderson ${ }^{2}$, Ming-Tseh Lin ${ }^{2}$, \\ Lisa Haley2, Ren-chin Wu2,3, Russell Vang ${ }^{2,4,5}$, Ie-ming Shih ${ }^{2,4,5}$, Robert J. Kurman ${ }^{2,4,5}$ \\ ${ }^{1}$ Department of Pathology, Sir Run Run Shaw Hospital, Zhejiang University School of Medicine, Hangzhou, 310016, China \\ ${ }^{2}$ Department of Pathology, Johns Hopkins Medical Institutions, Baltimore, 21231, MD, USA \\ ${ }^{3}$ Department of Pathology, Chang Gung Memorial Hospital and Chang Gung University College of Medicine, Taoyuan, 33378, \\ Taiwan \\ ${ }^{4}$ Department of Gynecology and Obstetrics, Johns Hopkins Medical Institutions, Baltimore, 21231, MD, USA \\ ${ }^{5}$ Department of Oncology, Johns Hopkins Medical Institutions, Baltimore, 21231, MD, USA
}

Correspondence to:

Robert J. Kurman, e-mail: rkurman@jhmi.edu

Keywords: ovarian, mucinous carcinoma, teratoma, microsatellite genotyping, HUMARA assay

Received: July 14, 2015

Accepted: August 20, 2015

Published: September 02, 2015

\section{ABSTRACT}

It is believed that a subset of primary ovarian mucinous tumors is derived from mature teratomas [1-5]. To confirm this, we performed microsatellite genotyping using a variety of short tandem repeat makers and analyzed allelotypes of 8 mucinous tumors ( 4 mucinous carcinomas, 3 atypical proliferative mucinous tumors and 1 mucinous cystadenoma) associated with a teratoma to determine whether they were clonally related. 7 of the $\mathbf{8}$ mucinous tumors showed complete or a high degree of homozygosity. Among the 6 pairs of tumors with teratoma tissue available for comparison, 5 of 6 showed a high or complete degree of allelotypes matching, which differed from the somatic allelotypes of the normal control tissue. A discrepancy was detected between carcinoma and teratoma in one pair at several loci, with different $X$-chromosome inactivation patterns revealed by the HUMARA clonality assay. We also investigated the allelotypes of $\mathbf{1 6}$ ovarian mucinous carcinomas without a teratoma in young patients (range 13-30) and in 6 older patients (range 40-67) using the same method. None of these tumors showed pure homozygosity. The number of homozygous loci in this cohort was significantly lower than that in the first. Our results suggest first, that most mucinous tumors associated with a teratoma are derived from the teratoma but occasionally they could be collision tumors and second that the majority of pure mucinous tumors in young women in whom a teratoma is not present are not derived from a teratoma.

\section{INTRODUCTION}

Ovarian mucinous tumors, including cystadenoma, atypical proliferative (borderline) mucinous tumor (APMT) and mucinous carcinoma, unlike other ovarian epithelial tumors, frequently occur in young women $[3,6]$. In addition, a subset of mucinous tumors is associated with teratomas and immunohistochemical studies showing that they have similar immunoprofiles supporting the interpretation that the mucinous tumors develop from mucinous epithelium in the teratoma [1-4]. Although these mucinous neoplasms are generally regarded as primary in the ovary the possibility of a metastasis from a colorectal carcinoma cannot be entirely excluded because their immunophenotypes can be identical [1-4]. To prove that this subset of mucinous tumors is derived from the associated teratoma, we performed microsatellite genotyping in 8 cases of combined mucinous tumors and teratomas and compared the allelotypes of each tumor to its normal control tissue at each short tandem repeats (STR) loci to determine whether or not the tumors were clonally-related.

We also speculated that mucinous tumors in young women in whom a teratoma was not detected may 
have arisen from a teratoma in which the teratomatous component was overgrown and obliterated by the mucinous neoplasm. However, little is known about zygosity of this entity, with the exception of one recent study investigating a cohort of patients ranging from 31-76 years, mean age 54.4[7], which showed heterozygosity in all the tumors. To test this hypothesis, we investigated the allelotypes of 16 ovarian mucinous carcinomas in young patients (13-30 years) and in 6 older patients (40-67 years) using the same method discussed above.

\section{RESULTS}

A cohort of 8 ovarian mucinous tumors ( 4 mucinous carcinomas, 3 APMTs and 1 mucinous cystadenoma) associated with mature cystic teratomas were included in this study (Table 1, Figure 1). The mean patient age was 35 years (range 16-57). Except for one mucinous carcinoma that was bilateral all the other tumors were unilateral. The two tumor components were of admixed on gross examination in 4 of the 8 cases while in the remaining 4 cases the tumors were close to one another but distinctly separate. In 3 cases bland mucinous epithelium was present in the teratoma (case 2, 4 and 6). Five of the 8 mucinous tumors ( 3 carcinomas and 2 APMT) in this cohort displayed pseudomyxoma ovarii. Another cohort of 22 ovarian mucinous carcinomas without a teratomatous component was also analyzed (Table 2, mean age 30.6; range 13-67), among which 16 patients were 30-years-old or younger (range 13-30). None of the tumors in this group were associated with pseudomyxoma ovarii. All the mucinous tumors in this study displayed an intestinal phenotype and there was no evidence of extra-ovarian disease.
Detailed microsatellite genotyping results at 9 tested loci are shown in Table 3 . In the cohort of 8 mucinous tumors associated with teratoma there were only 6 in which teratomatous tissue was available for analysis. Tumors 1-5 showed completely matching allelotypes with the corresponding teratomatous components. In this group, tumors 1, 2, 3 and 5 showed complete isodisomy at all informative loci. In tumors 6 and 7, the carcinoma samples also displayed a high degree of isodisomy at the informative loci $(100 \% ; 80 \%)$ although the teratoma DNA was not available for comparison. None of the 8 mucinous tumors were completely heterozygous at the 9 loci tested. In total (pairs1-7), 92.5\% (37/40) of informative loci were homozygous, the mean number of homozygous loci was 5.29 (range 4-6). The capillary electropherogram of two representative cases of mucinous carcinomas were shown in Figure 2. In pair 8, discrepancy at 4 STR loci was identified between the teratoma and carcinoma components (Figure 3). At the locus of D18S51 and FGA, different single alleles were present between the two tumors. The position of the two adjacent small peaks at D18S51 in the carcinoma overlapped with that of the long allele in control tissue, denoting a STR "biallelic" artifact most commonly reported at this locus, suggesting a possible somatic mutation [8]. The teratoma was homozygous at D13S317 while the carcinoma was heterozygous. The teratoma was heterozygous at TPOX locus while carcinoma was homozygous. The allelotypes of the carcinoma samples from both ovaries completely matched. In addition, there were no matching homozygous loci detected between the 2 tumors. Further the HUMARA clonality assay of the mucinous carcinoma and teratoma samples revealed different $\mathrm{X}$-chromosome inactivation (XCI) patterns between the teratoma and carcinomas while the carcinoma involving both ovaries shared the

\section{Table 1: Clinical and pathological features of mucinous tumors associated with mature cystic teratoma

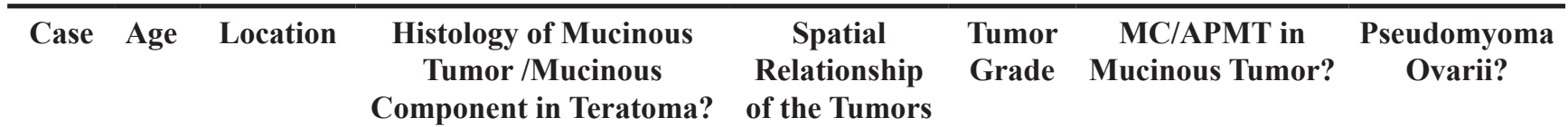

\begin{tabular}{|c|c|c|l|l|c|c|c|}
\hline 1 & 16 & RO & MC/N & Admixed & N/A & N/A & N \\
\hline 2 & 57 & RO & APMT/Y & Admixed & N/A & N/A & Y \\
\hline 3 & 57 & LO & APMT/N & Adjacent & N/A & N/A & Y \\
\hline 4 & 30 & RO & APMT/Y & Admixed & N/A & N/A & N \\
\hline 5 & 32 & LO & Carcinoma/N & Adjacent & 1 & Y & Y \\
\hline 6 & 28 & LO & Carcinoma/Y & Admixed & 1 & Y & Y \\
\hline 7 & 46 & RO & Carcinoma/N & Adjacent & 2 & Y & Y \\
\hline 8 & 23 & BO & Carcinoma//N & Adjacent & 2 & Y & N \\
\hline
\end{tabular}

LO: Left ovary; RO: Right ovary; BO: Bilateral ovary; MC: Mucinous cystadenoma; APMT: Atypical proliferative mucinous tumor; Y: Yes; N: No; N/A: Not applicable. 


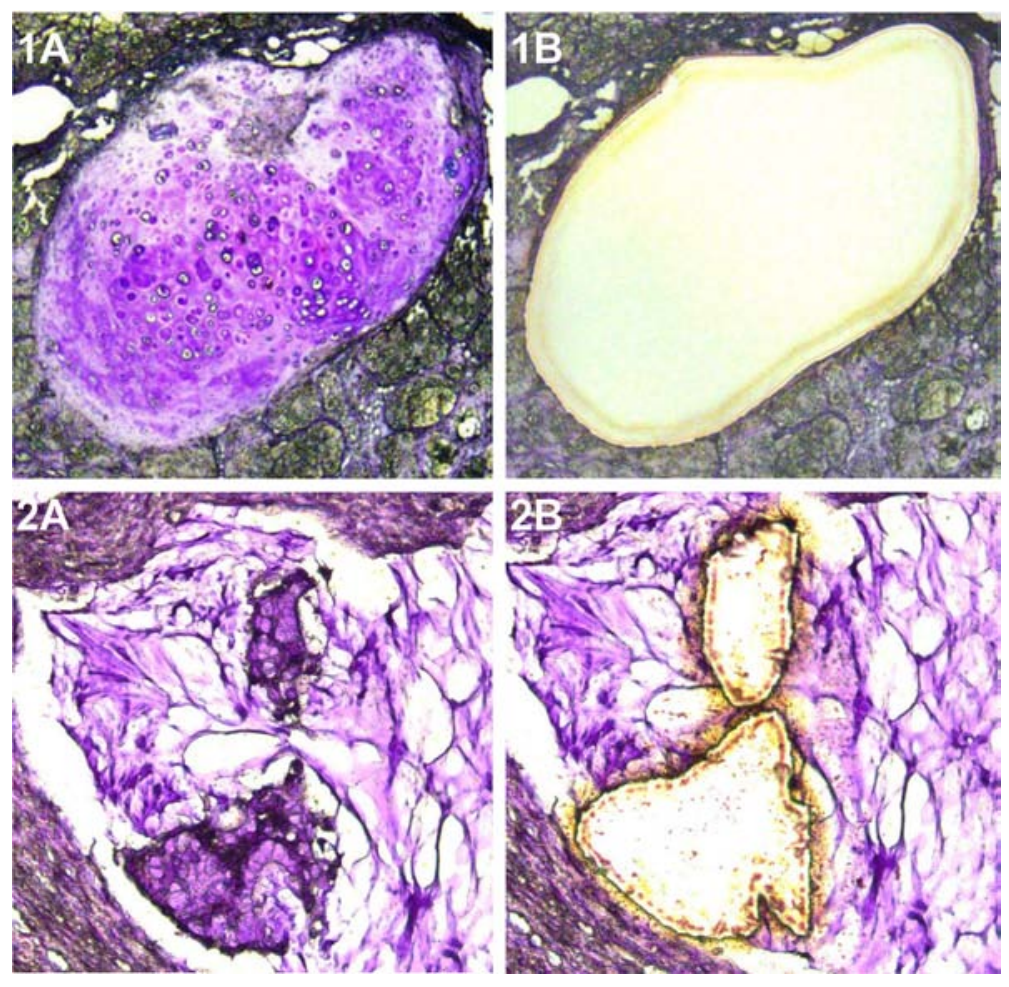

Figure 1: Microdissected teratoma and mucinous carcinoma component analyzed. A. Cartilagenous tissue dissected from mature cystic teratoma. B. Ruptured mucinous carcinomatous gland embedded in acellular mucin pool observed in a mucinous carcinoma associated with a teratoma.

same pattern (Figure 3A-H, Figure 3B-H, Figure 3C-H and Figure 3D-H). The corrected cleavage ratio of the teratoma sample was 3.03 , indicating a significant reduction $(>70 \%)$ of the short allele and predominance of the long allele, while in the carcinoma samples from both ovaries only the short alleles were present.

In the cohort of mucinous carcinoma without a teratoma, no purely homozygous carcinoma was detected in either the young (carcinoma 1-16) or older patients (carcinoma 17-22). Ten out of $16(62.5 \%)$ tumors in the group of young women and 3 out of the $6(50 \%)$ in the older group of women were completely heterozygous. In the total of 22 cases of mucinous carcinomas within this cohort, $13 \%(18 / 139)$ of informative loci were homozygous, the mean number of homozygous loci was 0.91 (range 0-3). The number of homozygous loci between young and elder patients in this cohort was not significantly different $(p=0.20)$. However, the number of homozygous loci between cohorts of mucinous tumors unassociated and associated with teratoma was significantly different $(p<0.01)$.

\section{DISCUSSION}

Clinicopathologic and molecular genetic studies have shown that most epithelial ovarian carcinomas develop from precursor lesions that are müllerian derived which is consistent with their müllerian phenotype. Accordingly, serous tumors are thought to develop from precursor lesions in the fallopian tube and it is generally accepted that endometrioid, clear cell and seromucinous tumors arise from endometriosis [9-14]. The pathogenesis of mucinous carcinoma, however, is not well established. It has been proposed that a subset arises from mature teratomas, $50-100 \%$ of which showing a CK7-/CK20+ phenotype [1, 5], would mimic metastatic colorectal carcinomas and therefore this can lead to difficulty in diagnosis of a primary versus a metastatic carcinoma. The presence of a teratomatous component is generally interpreted as evidence of ovarian origin, especially in young women with unilateral tumors. On the other hand, as mature teratomas are a common ovarian tumor in these individuals, it is conceivable that some are collision tumors.

In the majority of teratoma-associated mucinous tumors in this study, the complete or high degree of homozygosity and concordance in zygosity with the teratomatous components, is consistent with the findings of Kerr et al. and Fujii et al. [7, 15] and supports their germ-cell origin. However, in case 8, the 23-year-old patient with a unilateral teratoma and bilateral mucinous carcinoma, the discrepancy of allelotypes at loci of D18S51, FGA and D13S317 between the teratoma and carcinoma strongly suggests that the two tumors originated from different cellular clones although the 
Table 2: Clinical and pathological features of mucinous carcinoma unassociated with teratoma

\begin{tabular}{|c|c|c|c|c|c|}
\hline Case & Age & Site & Tumor Grade & MC/APMT present? & Pseudomyxoma ovarii? \\
\hline \multicolumn{6}{|l|}{ YP } \\
\hline 1 & 22 & $\mathrm{LO}$ & 1 & $\mathrm{Y}$ & $\mathrm{N}$ \\
\hline 2 & 22 & $\mathrm{LO}$ & 1 & $\mathrm{Y}$ & $\mathrm{N}$ \\
\hline 3 & 27 & LO & 1 & $\mathrm{~N}$ & $\mathrm{~N}$ \\
\hline 4 & 29 & LO & 1 & Y & $\mathrm{N}$ \\
\hline 5 & 27 & LO & 3 & $\mathrm{Y}$ & $\mathrm{N}$ \\
\hline 6 & 30 & LO & 2 & Y & $\mathrm{N}$ \\
\hline 7 & 16 & LO & 1 & $\mathrm{Y}$ & $\mathrm{N}$ \\
\hline 8 & 18 & RO & 1 & $\mathrm{Y}$ & $\mathrm{N}$ \\
\hline 9 & 24 & $\mathrm{LO}$ & 1 & $\mathrm{Y}$ & $\mathrm{N}$ \\
\hline 10 & 30 & RO & 1 & $\mathrm{Y}$ & $\mathrm{N}$ \\
\hline 11 & 29 & $\mathrm{LO}$ & 2 & $\mathrm{Y}$ & $\mathrm{N}$ \\
\hline 12 & 13 & LO & 2 & $\mathrm{~N}$ & $\mathrm{~N}$ \\
\hline 13 & 30 & LO & 1 & Y & $\mathrm{N}$ \\
\hline 14 & 15 & RO & 2 & Y & $\mathrm{N}$ \\
\hline 15 & 28 & RO & 2 & $\mathrm{~N}$ & $\mathrm{~N}$ \\
\hline 16 & 27 & LO & 2 & $\mathrm{~N}$ & $\mathrm{~N}$ \\
\hline \multicolumn{6}{|l|}{$\mathrm{OP}$} \\
\hline 17 & 58 & LO & 1 & $\mathrm{Y}$ & $\mathrm{N}$ \\
\hline 18 & 47 & $\mathrm{LO}$ & 1 & $\mathrm{~N}$ & $\mathrm{~N}$ \\
\hline 19 & 50 & RO & 1 & $\mathrm{Y}$ & $\mathrm{N}$ \\
\hline 20 & 54 & $\mathrm{RO}$ & 1 & $\mathrm{~N}$ & $\mathrm{~N}$ \\
\hline 21 & 40 & LO & 1 & Y & $\mathrm{N}$ \\
\hline 22 & 67 & LO & 2 & $\mathrm{Y}$ & $\mathrm{N}$ \\
\hline
\end{tabular}

MC: Mucinous cystadenoma; APMT: Atypical proliferative mucinous tumor; YP: Young patients; OP: Older patients; LO: Left ovary; RO: Right ovary; Y: Yes; N: No.

difference at the locus of TPOX could be a result of loss of heterozygosity (LOH). The different XCI patterns revealed by the HUMARA clonality assay also supports the interpretation that the tumors are not genetically related while the concordant XCI pattern between the bilateral tumors is consistent with their shared clonality [16-22]. Although there was no evidence of carcinoma elsewhere, this case could nonetheless represent a metastatic mucinous carcinoma from an occult primary [23-25], especially considering the fact that the tumors were bilateral. Another possibility, although much less likely in the absence of extra-ovarian disease, is that the carcinoma originated from one ovary, and was associated with a teratoma (a collision tumor) and metastasized to the other ovary.
There was no support for our hypothesis that mucinous tumors in young women that were not associated with a teratoma could have arisen from a teratoma that was subsequently obliterated by an expanding mucinous neoplasm. The finding was consistent with Kerr's study [7], which analyzed 9 cases of intestinal type ovarian mucinous carcinoma without teratoma, however only in an older patient group (mean age 55.4, range 31-76 years old). The majority of the tumors in this cohort showed heterozygous allelotypes, which is consistent with the somatic cell allelotype of the normal control tissue. The 1-3 homozygous loci in each case identified in 9 of 22 cases could be explained by $\mathrm{LOH}$, a frequent molecular event in carcinoma. Combining Kerr's study with ours[7], in a total of 
Table 3: Microsatellite genotyping of ovarian mucinous tumor at 9 Microsatellite loci

\begin{tabular}{|c|c|c|c|c|c|c|c|c|c|c|}
\hline \multicolumn{2}{|l|}{ Case } & \multirow{2}{*}{$\frac{\text { D3S1358 }}{\square}$} & \multirow{2}{*}{$\frac{\text { vWA }}{n}$} & \multirow{2}{*}{$\frac{\text { FGA }}{n}$} & \multirow{2}{*}{ 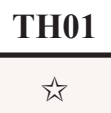 } & \multirow{2}{*}{$\frac{\text { TPOX }}{\square}$} & \multirow{2}{*}{$\frac{\text { CSF1PO }}{\square}$} & \multirow{2}{*}{$\frac{\text { D5S818 }}{\text { 质 }}$} & \multirow{2}{*}{$\frac{\text { D13S317 }}{\text { 质 }}$} & \multirow{2}{*}{$\begin{array}{c}\text { D7S820 } \\
\square\end{array}$} \\
\hline Doiry & $\mathrm{MC}$ & & & & & & & & & \\
\hline rail & Teratoma & घ & $\square$ & $\square$ & 必 & $\square$ & घ & 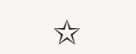 & 必 & घ \\
\hline \multirow[t]{2}{*}{ Pair2 } & APMT & $\mathbf{\square}$ & $\mathbf{\square}$ & $\mathbf{\square}$ & $\Delta$ & 颈 & $\mathbf{\square}$ & 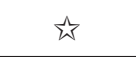 & $\mathbf{\square}$ & 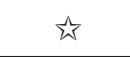 \\
\hline & Teratoma & 口 & $\mathbf{\square}$ & $\mathbf{\square}$ & 必 & 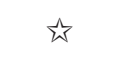 & $\mathbf{\square}$ & $\sqrt{2}$ & $\square$ & 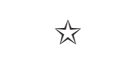 \\
\hline \multirow{2}{*}{ Pair3 } & APMT & 口 & 口 & - & $\bullet$ & 口 & $\sqrt{2}$ & $\bullet$ & 口 & - \\
\hline & Teratoa & $\mathbf{\square}$ & $\mathbf{\square}$ & $\mathbf{\square}$ & $\bullet$ & $\mathbf{\square}$ & 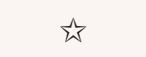 & $\bullet$ & $\mathbf{\square}$ & - \\
\hline \multirow{2}{*}{ Pair4 } & APMT & घ & $\mathbf{\square}$ & - & 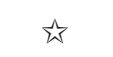 & 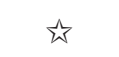 & $\iota^{2}$ & घ & $\mathbf{\square}$ & $\sqrt{2}$ \\
\hline & Teratoma & घ & $\mathbf{\square}$ & घ & 头 & 放 & 就 & $\square$ & घ & 功 \\
\hline \multirow{2}{*}{ Pair5 } & Carcinoma & घ & घ & घ & 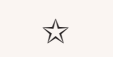 & $\sqrt{x}$ & 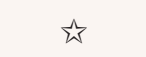 & घ & - & घ \\
\hline & Teratoma & $\square$ & $\square$ & - & 功 & 功 & 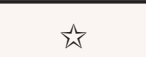 & $\square$ & $\square$ & 口 \\
\hline \multirow{2}{*}{ Pair6 } & Carcinoma & घ & 口 & 头 & $\sqrt{2}$ & 口 & 次 & $\square$ & $\square$ & घ \\
\hline & Teratoma & - & - & - & - & - & - & - & - & - \\
\hline \multirow{2}{*}{ Pair7 } & Carcinoma & & $\bullet$ & घ & 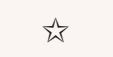 & 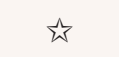 & - & $\sqrt{2}$ & - & घ \\
\hline & Teratoma & - & - & - & - & - & - & - & - & - \\
\hline \multirow{3}{*}{ Pair8 } & $\begin{array}{l}\text { Carcinoma } \\
\text { (1) }\end{array}$ & $\bullet$ & $\bullet$ & $\Delta$ & $\bullet$ & $\mathbf{\square}$ & $\bullet$ & $\sqrt{x}$ & - & $\bullet$ \\
\hline & $\begin{array}{l}\text { Carcinoma } \\
\text { (r) }\end{array}$ & $\bullet$ & $\bullet$ & $\Delta$ & $\bullet$ & $\mathbf{\square}$ & $\bullet$ & $\Delta$ & $\bullet$ & $\bullet$ \\
\hline & $\begin{array}{l}\text { Teratoma } \\
(\mathrm{r})\end{array}$ & $\bullet$ & $\bullet$ & घ & $\bullet$ & $\bullet$ & $\bullet$ & 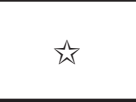 & $\mathbf{\square}$ & $\bullet$ \\
\hline \multicolumn{2}{|c|}{ Carcinoma 1} & 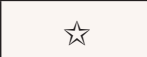 & 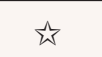 & 勾 & $\bullet$ & - & 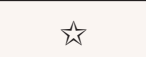 & $\bullet$ & - & $\bullet$ \\
\hline \multicolumn{2}{|c|}{ Carcinoma 2} & $\bullet$ & 为 & $\Delta$ & $\bullet$ & $\bullet$ & $\bullet$ & $\bullet$ & $\bullet$ & $\bullet$ \\
\hline \multicolumn{2}{|c|}{ Carcinoma 3} & 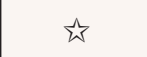 & $\bullet$ & $\bullet$ & $\bullet$ & 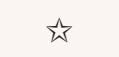 & $\bullet$ & $\bullet$ & $\bullet$ & $\sqrt{2}$ \\
\hline \multicolumn{2}{|c|}{ Carcinoma 4} & $\bullet$ & $\square$ & $\bullet$ & $\bullet$ & $\bullet$ & $\bullet$ & - & $\mathbf{0}$ & - \\
\hline \multicolumn{2}{|c|}{ Carcinoma 5} & 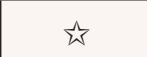 & $\bullet$ & 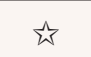 & $\bullet$ & - & - & $\bullet$ & $\Sigma$ & $\bullet$ \\
\hline \multicolumn{2}{|c|}{ Carcinoma 6} & $\bullet$ & 放 & $\bullet$ & $\bullet$ & 次 & 就 & 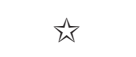 & $\mathbf{\square}$ & - \\
\hline \multicolumn{2}{|c|}{ Carcinoma 7} & $\bullet$ & $\bullet$ & $\bullet$ & $\bullet$ & $\sqrt{x}$ & ț & $\bullet$ & $\bullet$ & $\sqrt{x}$ \\
\hline \multicolumn{2}{|c|}{ Carcinoma 8} & $\bullet$ & $\square$ & - & - & $\bullet$ & 次 & 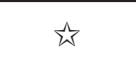 & - & $\bullet$ \\
\hline \multicolumn{2}{|c|}{ Carcinoma 9} & $\bullet$ & - & - & $\bullet$ & - & $\bullet$ & 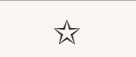 & $\bullet$ & - \\
\hline \multicolumn{2}{|c|}{ Carcinoma 10} & 必 & 口 & $\bullet$ & घ & $\sqrt{x}$ & $\bullet$ & 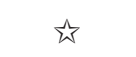 & $\bullet$ & घ \\
\hline Carci & a 11 & $\sqrt{x}$ & $\sqrt{2}$ & - & 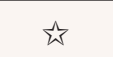 & 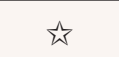 & $\sqrt{x}$ & $\bullet$ & $\bullet$ & - \\
\hline Carci & a 12 & $\bullet$ & $\bullet$ & $\bullet$ & $\bullet$ & $\bullet$ & $\bullet$ & $\bullet$ & $\bullet$ & $\bullet$ \\
\hline Carci & a 13 & th & - & 口 & - & $\bullet$ & $\bullet$ & $\bullet$ & $\bullet$ & - \\
\hline Carci & 14 & 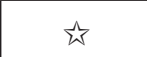 & $\bullet$ & $\bullet$ & $\bullet$ & $\square$ & - & • & $\bullet$ & - \\
\hline Carci & a 15 & 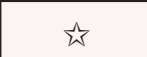 & • & - & - & $\vec{k}$ & 口 & 口 & 口 & - \\
\hline
\end{tabular}

(Continued) 


\begin{tabular}{|c|c|c|c|c|c|c|c|c|c|}
\hline Case & D3S1358 & vWA & FGA & TH01 & TPOX & CSF1PO & D5S818 & D13S317 & D7S820 \\
\hline Carcinoma 16 & $\bullet$ & $\bullet$ & $\sqrt{x}$ & 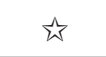 & $\bullet$ & $\hbar$ & $\bullet$ & $\bullet$ & 一 \\
\hline Carcinoma 17 & घ & $\bullet$ & $\bullet$ & - & 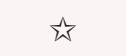 & 一 & $\bullet$ & $\bullet$ & $\bullet$ \\
\hline Carcinoma 18 & - & tris & trit & 在 & - & - & $\bullet$ & $\bullet$ & th \\
\hline Carcinoma 19 & $\bullet$ & $\bullet$ & $\sqrt{3}$ & $\bullet$ & $\bullet$ & $\bullet$ & $\bullet$ & $\bullet$ & 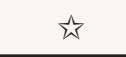 \\
\hline Carcinoma 20 & $\bullet$ & 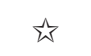 & 项 & $\bullet$ & - & - & 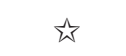 & $\bullet$ & - \\
\hline Carcinoma 21 & $\sqrt{s}$ & $\bullet$ & $\bullet$ & $\bullet$ & $\bullet$ & $\bullet$ & 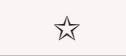 & $\bullet$ & $\bullet$ \\
\hline Carcinoma 22 & - & • & • & - & • & $\sqrt{T}$ & $\bullet$ & • & 幽 \\
\hline
\end{tabular}

APMT: Atypical proliferative mucinous tumor; MC: Mucinous cystadenoma; 1: left; r: right. Allelotypes of each tumor at 9 tested short tandem repeat loci are indicated as different shapes, with star indicating uninformative loci, square and triangle indicating homozygous loci with different allele present, circle indicating heterozygous loci and hyphen indicating loci failed to PCR amplify. Loci were considered heterozygous when the 2 alleles in the tumor tissue matched 2 alleles in the control tissue. In contrast, loci were considered homozygous when 1 allele was seen in tumor tissue while 2 alleles were seen in control tissue. Uninformative loci represented those with only one allele present in the normal control.

31 cases of ovarian mucinous carcinoma, 16 young and 15 older, the carcinomas in the young women did not show significant differences in terms of homozygous loci numbers compared to the older women $(p=0.26)$, although the number of cases is still relatively small. It is therefore likely that these carcinomas developed from a non-germ cell precursor lesion. It should be noted that teratomas can demonstrate various degrees of host isodisomy, depending on their underlying meiotic errors and molecular genetic makers used for testing [7, 15, 26-30]. For example, the teratoma in case 8 displayed only 2 homozygous loci among 8 informative loci included in the AmpFLSTR Prifiler kit. This partial homozygosity could be explained either by contamination of somatic tissue or suppression of meiosis II following meiosis I, leading to the development of a teratoma [26-30]. In fact, a minority of teratomas generated from meiosis I failure could be of identical zygosity of the host (somatic) tissue [28-30]. We favor the latter explanation as pure sebaceous gland tissue, devoid of lymphocytes, were laser capture micro-dissected and used for this case to extract DNA. It is thus conceivable that a carcinoma derived from the teratoma would also display 2 homozygous loci at the most, if there were no LOH. Therefore, the possibility of germ-cell origin of some, if not all, of the tumors in this cohort could not be completely excluded by this method.

In addition, the high prevalence of pseudomyxoma ovarii $(71.4 \%, 5$ out of 7$)$ in atypical proliferative mucinous tumors and mucinous carcinomas associated with teratomas in our study (Case 1-7) and complete absence in those not associated with a teratoma suggests that pseudomyxoma ovarii in association with a unilateral mucinous carcinoma or atypical proliferative mucinous tumor may originate from a teratoma. If not detected initially, additional sections can be performed in an effort to detect a teratoma.
In conclusion, our study shows that most mucinous tumors associated with a teratoma are derived from the teratoma but that on occasion they may be collision tumors. Furthermore, ovarian mucinous carcinomas unassociated with teratoma in both young and older women mostly likely are of nongerm cell in origin, however, our numbers were relatively small and therefore study of more cases using different types of molecular analysis is necessary to definitively support this interpretation.

\section{MATERIALS AND METHODS}

\section{Tissue specimen collection}

We retrieved formalin-fixed paraffin-embedded (FFPE) tissue blocks from 8 ovarian mucinous tumors associated with teratoma and 22 cases of ovarian mucinous carcinoma not associated with a teratoma from the consult and in-house surgical pathology archives of The Johns Hopkins Hospital. Slides were reviewed by three gynecologic pathologists (LES, RV and RJK). A total of 34 FFPE blocks was collected from the tumors and 30 FFPE blocks from fallopian tubes, which served as normal controls. The study was approved by the Institutional Review Board of The Johns Hopkins Hospital.

\section{Laser capture microdissection, macrodissection and DNA extraction}

Twelve micron-thick sections were obtained from FFPE tissue blocks of the tumors, placed on membrane slides (Carl Zeiss MicroImaging, Göttingen, Germany) and counterstained with hematoxylin. Tumour cells from mucinous neoplastic epithelium and teratoma were microdissected using the PALM laser capture 
2A-1

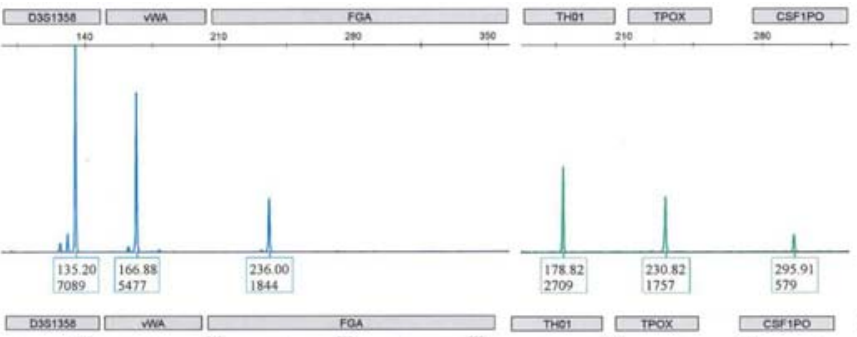

2A-2
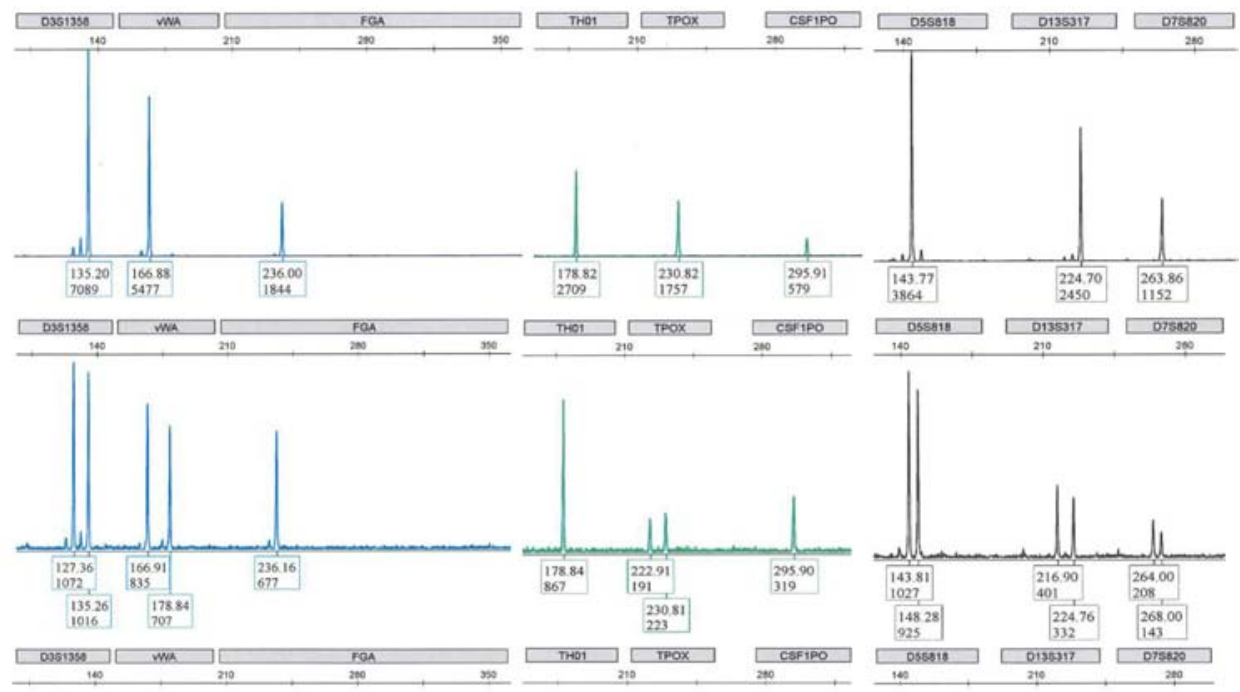

2B-1
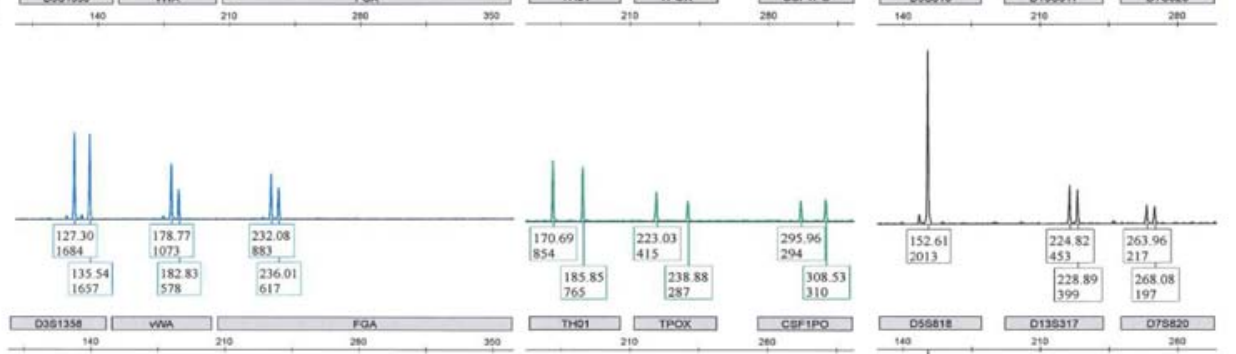

2B-2
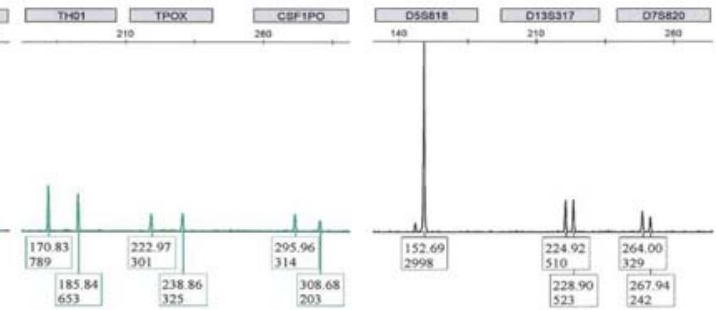

Figure 2: Allelotypes of representative cases of mucinous carcinomas at 9 STR loci are shown in capillary electropherogram. The locus with a heterozygous pattern in the control tissue was considered informative. Upper panel: Mucinous carcinoma 2A-1. associated with teratoma and normal fallopian tube 2A-2. The carcinoma sample displayed homozygosity at all 6 informative loci (D3S1358, vWA, TPOX, D5S818, D13S317 and D7S820) while the rest 3 loci were homozygous in control (fallopian tube) tissue and considered uninformative. Lower panel: Mucinous carcinoma 2B-1. unassociated with teratomas and normal fallopian tube 2B-2. The carcinoma sample showed heterozygosity at all eight informative loci (D3S1358, vWA, FGA, TH01, TPOX, CSF1PO, D13S317 and D7S820).

microdissection microscope (Leica Microsistem, LMD 7000). For normal control tissue, 10 micron-thick sections of fallopian tube tissue were obtained from the FFPE blocks. After $24 \mathrm{~h}$ of proteinase $\mathrm{K}$ digestion, genomic DNA was extracted using a QIAamp DNA FFPE Kit (Qiagen, Valencia, CA) following the vendor's protocol.

\section{Microsatellite genotyping analysis}

Nine microsatellite loci (D3S1358, vWA, FGA, TH01, TPOX, CSF1PO, D5S818, D13S317 and D7S820) were PCR amplified using AmpFLSTR Prifiler kit (Applied Biosystems, Foster City, CA) in the 8 ovarian mucinous tumors associated with mature teratoma and 22 cases of ovarian mucinous carcinoma. 6 additional loci (D8S1179, D21S11, D7S820, D16S539, D2S1338 and D19S433) were tested in case 8 in this cohort using AmpFLSTR Identifiler kit (Applied Biosystems, Foster City, CA). The following thermocycling conditions were used: initial denaturation at $95^{\circ} \mathrm{C}$ for 1 minute, followed by 28 cycles at 94 for 1 minute, $59^{\circ} \mathrm{C}$ for 1 minute, and $72^{\circ} \mathrm{C}$ for 1 minute, followed by a final extension at $60^{\circ} \mathrm{C}$ for $45 \mathrm{~s}$. After multiplex PCR amplification, $1 \mu \mathrm{l}$ of PCR product was mixed with $9 \mu \mathrm{l}$ of HiDi formamide/GeneScan 500 [ROX] size standard mixture (Applied Biosystems, Foster City, CA, USA). Fragments sizes were analyzed on the ABI3130xl Genetic Analyzer (Applied Biosystems, Foster City, CA, USA). Each locus displaying 2 major peaks were considered heterozygous and homozygous if only 1 major peak was shown. The genotype of each tumor was compared to that of normal control tissue for each of the loci tested. Only the locus with a heterozygous pattern in the control tissue was considered informative. The locus was considered uninformative if the control tissue displayed a homozygous allelotype or if the amplicon was insufficient for a certain allele size call. 

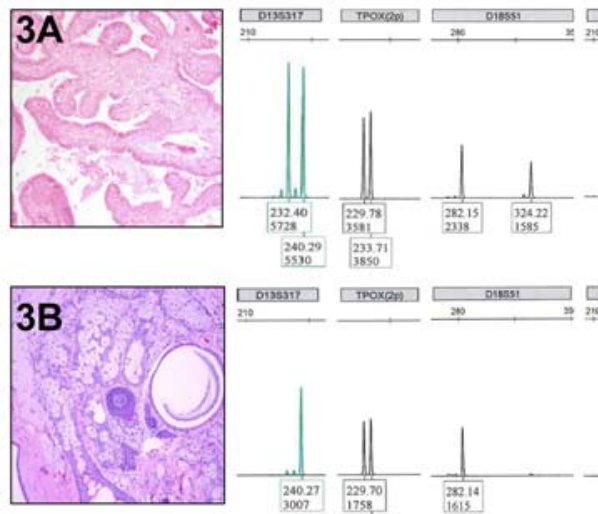

orsesm

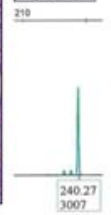

Troxan.

${ }_{2 \times 23}^{24650}$
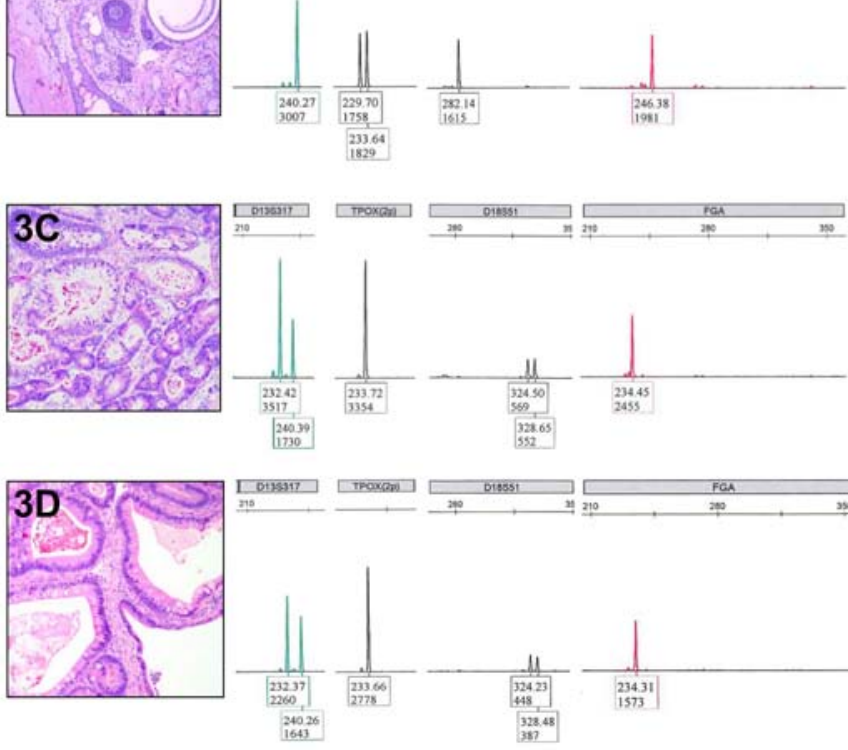

Troxper
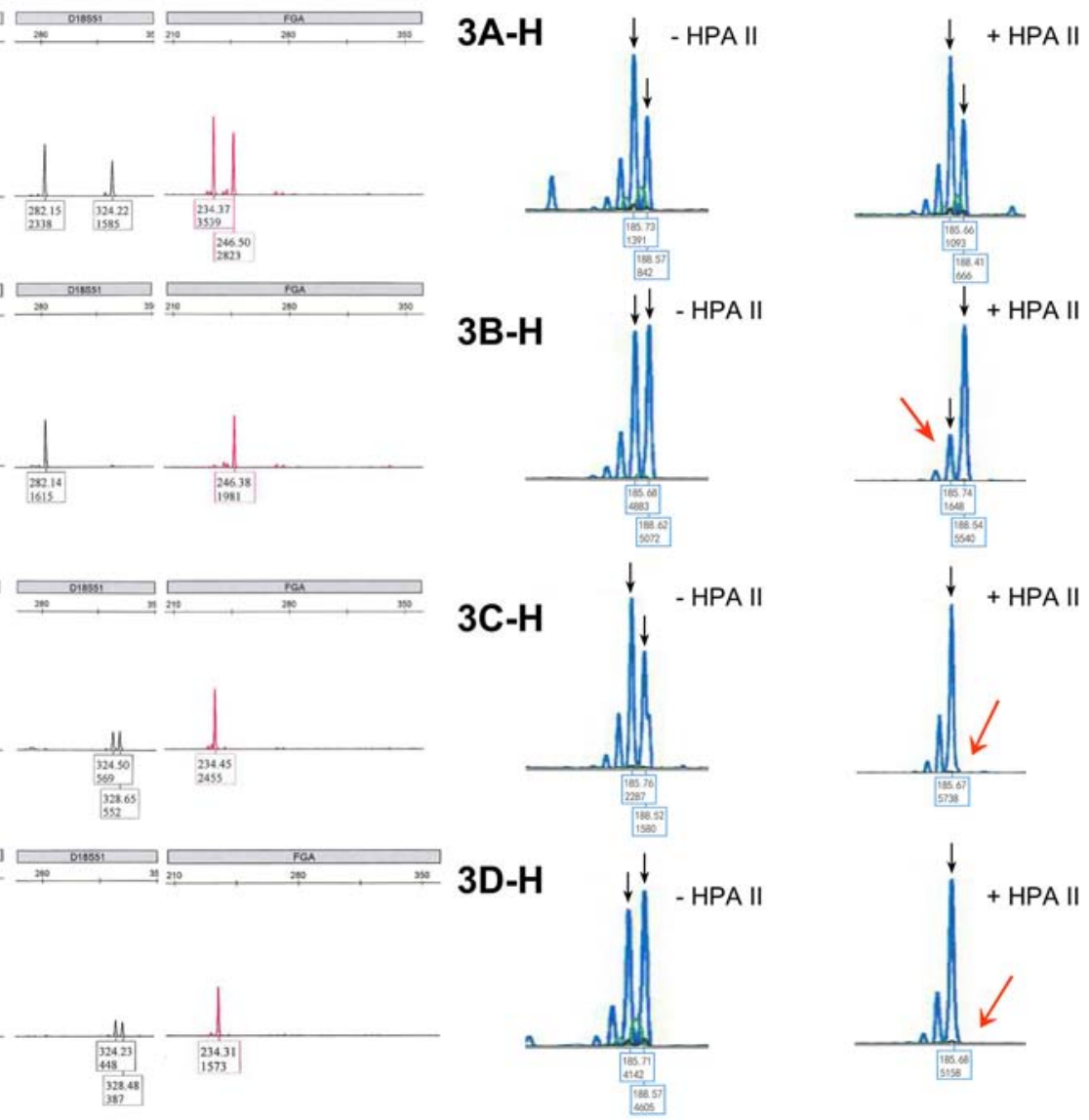

Figure 3: Allelotypes of different tumors at representative STR Loci and HUMARA assay of case 8. Fallopian tube 3A. teratoma in the right ovary 3B. mucinous carcinoma from left 3C. and right ovary 3D. were dissected to extract DNA. Left panel: capillary electropherogram showed that at the loci of D18S51 and FGA, different single alleles were present between the two tumors (3B, 3C, 3D) while the control tissue sample showed two alleles (3A). The two adjacent small peaks in the two carcinomas from bilateral ovaries denoted a STR "biallelic" artifact, suggesting a possible somatic mutation. The teratoma (3B) was homozygous at D13S317 while carcinoma (3C, 3D) was heterozygous. The teratoma (3B) was heterozygous at TPOX locus while carcinoma (3C, 3D) was the homozygous. The allelotypes of carcinoma samples from both ovaries (3C, 3D) completely matched. HUMARA assay showing different XCI patterns between teratoma and mucinous carcinoma 3A-H, 3B-H, 3C-H. and 3D-H. The electropherogram shows the fragment analysis of PCR products amplified from undigested and digested DNA of control tissue $(3 \mathrm{~A}-\mathrm{H})$, teratoma $(3 \mathrm{~B}-\mathrm{H})$ and carcinoma from both sides $(3 \mathrm{C}-\mathrm{H}$, 3D-H). 2 major peaks represented 2 alleles with different numbers of short tandem repeats at the HUMARA locus (black arrows). After digestion, the control DNA retained a balanced XCI pattern (3A-H), teratoma sample displayed preferential loss of the short allele (3B-H, red arrow) and carcinoma samples showed complete loss of the long alleles(3C-H, 3D-H, red arrows).

\section{HUMARA ASSAY (DNA digestion and PCR)}

HUMARA assay was performed in pair 8 by using the following protocol: 5-10 $\mu \mathrm{l}$ DNA from each tumor and control tissue were incubated for 16 hours at $37^{\circ} \mathrm{C}$ in a $25 \mu \mathrm{l}$ reaction containing $10 \mathrm{U}$ of the methylationsensitive restriction enzyme HpaII (New England Biolabs, Ipswich, MA). In parallel, each sample was subjected to mock digestion in buffer devoid of restriction enzyme. The reaction was terminated by incubation at $80^{\circ} \mathrm{C}$ for 20 minutes according to the manufacturer's instructions, followed by purification using QIAquick PCR Purification Kit (Qiagen, Valencia, CA). Both digested and mockdigested DNAs were PCR amplified (Applied Biosystems, Foster City, CA) using the following thermocycling conditions: initial denaturation at $94^{\circ} \mathrm{C}$ for 30 s, followed by 40 cycles at 94 for $30 \mathrm{~s}, 62^{\circ} \mathrm{C}$ for $45 \mathrm{~s}$, and $68^{\circ} \mathrm{C}$ for $60 \mathrm{~s}$. All PCRs were finally elongated at $68^{\circ} \mathrm{C}$ for $5 \mathrm{~min}$. The amplification primers used for HUMARA locus were as follows: 5'-TCCAGAATCTGTTCCAGAGCGTGC-3' (sense) and 5'-CTCTACGATGGGCTTGGGGAGAAC-3' (antisense). The antisense primer was labeled with 6FAM at the $5^{\prime}$ end. Capillary electrophoresis of the PCR products was performed on an ABI 3130 XL Genetic Analyzer Sequencer (Applied Biosystems, Foster City, CA) and fragment sizes were analyzed with the use of GeneMapper Software vs 5 (Applied Biosystems, Foster City, CA). The patient was considered heterozygous and informative when the PCR of undigested DNA from the control tissue showed two major peaks, representing the maternal and 
paternal alleles respectively. To compensate for possible preferential amplification of the shorter allele, we calculated $\mathrm{CR}_{\text {tumor }}$ (cleavage ratio of tumor) and $\mathrm{CR}_{\text {ctrl }}$ (cleavage ratio of control tissue) by using the following formula: (peak1 height of undigested sample $\div$ peak 2 height of undigested sample) $\div$ (peak 1 height of digested sample -peak 2 height of digested sample) [13-14]. To compensate for possible constitutional $\mathrm{XCI}$ skewing, $\mathrm{CR}_{\text {cor }}$ (corrected cleavage ratio of tumor) was calculated using the following formula: $\mathrm{CR}_{\text {tumor }} \div \mathrm{CR}_{\text {ctrl }}$ [14]. $\mathrm{CR}_{\text {cor }}$ of more than 2 or less than 0.5 , representing a preferential loss of intensity of $50 \%$ of one of the two alleles in the digested DNA was determined as predominance of one single allele and thus a monoclonal pattern [14]. A discrepancy in XCI pattern represents different clone origins between different tumors while a matching XCI pattern indicates a $1 / 2$ chance of shared origin.

\section{Statistical analysis}

Statistical analysis was performed using paired $t$ test in GraphPad Prisma 5.0.

\section{ACKNOWLEDGMENTS}

This study was in part supported by Sir Run Run Shaw Hospital, Zhejiang University (Young Investigator Research Fund), Hangzhou, China. YW was supported by physician rotation program of Sir Run Run Shaw Hospital, Zhejiang University, Hangzhou, China.

\section{CONFLICTS OF INTEREST} declare.

The authors don't have conflicts of interest to

\section{Author contributions}

RJK, YW, and LES conceived the project. YW, DA and LH carried out the experiment. YW, RCW and MTL were involved in data interpretation. LES, RV and RJK provided/ pathologically reviewed the cases in the study. YW, IES and RJK wrote/revised the manuscript.

\section{REFERENCES}

1. Vang R, Gown AM, Zhao C, Barry TS, Isacson C, Richardson MS, Ronnett BM. Ovarian mucinous tumors associated with mature cystic teratomas: morphologic and immunohistochemical analysis identifies a subset of potential teratomatous origin that shares features of lower gastrointestinal tract mucinous tumors more commonly encountered as secondary tumors in the ovary. Am J Surg Pathol. 2007; 31:8548-8569.
2. Hristov AC, Young RH, Vang R, Yemelyanova AV, Seidman JD, Ronnett BM. Ovarian metastases of appendiceal tumors with goblet cell carcinoidlike and signet ring cellpatterns:a report of 30 cases. Am J Surg Pathol. 2007; 31:1502-1511.

3. Soslow RA. Mucinous ovarian carcinoma: slippery business. Cancer. 2011; 117:451-453.

4. Black JD, Roque DM, Pasternak MC, Buza N, Rutherford TJ, Schwartz PE, McCarthy S, Ratner E. A series of malignant ovarian cancers arising from within a mature cystic teratoma: a single institution experience. Int J Gynecol Cancer. 2015; 25:792-797.

5. McKenney JK, Soslow RA, Longacre TA. Ovarian mature teratomas with mucinous epithelial neoplasms: morphologic heterogeneity and association with pseudomyxoma peritonei. Am J Surg Pathol. 2008; 32:645-655.

6. Young RH. Ovarian tumors and tumor-like lesions in the first three decades. Semin Diagn Pathol. 2014; 31:382-426.

7. Kerr SE, Flotte AB, McFalls MJ, Vrana JA, Halling KC, Bell DA. Matching maternal isodisomy in mucinous carcinomas and associated ovarian teratomas provides evidence of germ cell derivation for some mucinous ovarian tumors. Am J Surg Pathol. 2013; 37:1229-1235.

8. Clayton TM, Guest JL, Urquhart AJ, Gill PD. A genetic basis for anomalous band patterns encountered during DNA STR profiling. J Forensic Sci. 2004; 49:1207-1214.

9. Nik NN, Vang R, Shih IeM, Kurman RJ. Origin and pathogenesis of pelvic (ovarian, tubal, and primary peritoneal) serous carcinoma. Annu Rev Pathol. 2014; 9:27-45.

10. Anglesio MS, Bashashati A, Wang YK, Senz J, Ha G, Yang W, Aniba MR, Prentice LM, Farahani H, Li Chang H, Karnezis AN, Marra MA, Yong PJ, et al. Multifocal endometriotic lesions associated with cancer are clonal and carry a high mutation burden. J Pathol. 2015; 236:201-209.

11. Kurman RJ, Shih IeM. The origin and pathogenesis of epithelial ovarian cancer: a proposed unifying theory. Am J Surg Pathol. 2010; 34:433-443.

12. Prowse AH, Manek S, Varma R, Liu J, Godwin AK, Maher ER, Tomlinson IP, Kennedy SH. Molecular genetic evidence that endometriosis is a precursor of ovarian cancer. Int J Cancer. 2006; 119:556-562.

13. Ness RB. Endometriosis and ovarian cancer: thoughts on shared pathophysiology. Am J Obstet Gynecol. 2003; 189:280-294.

14. Kurman RJ, Carcangiu ML, Herrington CS, Young RH. World Health Organization classification of Tumors of Female Reproductive Organs. Lyon, France: IARC Press (4th Edition) 2014.

15. Fujii K, Yamashita Y, Yamamoto T, Takahashi K, Hashimoto K, Miyata T, Kawai K, Kikkawa F, Toyokuni S, Nagasaka T. Ovarian mucinous tumors arising from mature cystic teratomas - a molecular 
genetic approach for understanding the cellular origin. Hum Pathol. 2014; 45:717-724.

16. Gu J, Roth LM, Younger C, Michael H, Abdul-Karim FW, Zhang S, Ulbright TM, Eble JN, Cheng L. Molecular evidence for the independent origin of extra-ovarian papillary serous tumors of low malignant potential. J Natl Cancer Inst. 2001; 93:1147-1152.

17. Shattuck TM, Westra WH, Ladenson PW, Arnold A. Independent clonal origins of distinct tumor foci in multifocal papillary thyroid carcinoma. N Engl J Med. 2005; 352:2406-2412.

18. Vogelstein B, Fearon ER, Hamilton SR, Preisinger AC, Willard HF, Michelson AM, Riggs AD, Orkin SH. Clonal analysis using recombinant DNA probes from the X-chromosome. Cancer Res. 1987; 47:4806-4813.

19. McCaw BK, Latt SA. X-chromosome replication in parthenogenic benign ovarian teratomas. Hum Genet. 1977; 38:253-264.

20. Martin GR, Epstein CJ, Travis B, Tucker G, Yatziv S, Martin DW Jr, Clift S, Cohen S. X-chromosome inactivation during differentiation of female teratocarcinoma stem cells in vitro. Nature. 1978; 271:329-333.

21. McMahon A, Fosten M, Monk M. Random X-chromosome inactivation in female primordial germ cells in the mouse. J Embryol Exp Morphol. 1981; 64:251-258.

22. Looijenga LH, Gillis AJ, van Gurp RJ, Verkerk AJ, Oosterhuis JW. X inactivation in human testicular tumors.
XIST expression and androgen receptor methylation status. Am J Pathol. 1997; 151:581-590.

23. Riihimäki M, Hemminki A, Sundquist K, Hemminki K. Causes of death in patients with extranodal cancer of unknown primary: searching for the primary site. BMC Cancer. 2014; 14:439.

24. Riihimäki M, Hemminki A, Sundquist K, Hemminki K. Time trends in survival from cancer of unknown primary: small steps forward. Eur J Cancer. 2013; 49:2403-2410.

25. Pentheroudakis G, Briasoulis E, Pavlidis N. Cancer of unknown primary site: missing primary or missing biology? Oncologist. 2007; 12:418-425.

26. Linder D, Power J. Further evidence for post-meiotic origin of teratomas in the human female. Ann Hum Genet. 1970; 34:21-30.

27. Dahl N, Gustavson KH, Rune C, Gustavsson I, Pettersson U. Benign ovarian teratomas. An analysis of their cellular origin. Cancer Genet Cytogenet. 1990; 46:115-123.

28. Parrington JM, West LF, Povey S. The origin of ovarian teratomas. J Med Genet. 1984; 21:4-12.

29. Surti U, Hoffner L, Chakravarti A, Ferrell RE. Genetics and biology of human ovarian teratomas I. Cytogenetic analysis and mechanism of origin. Am J Hum Genet. 1990; 47:635-643.

30. Sobis H, Verstuyf A, Vandeputte M. Endodermal origin of yolk-sac-derived teratomas. Development. 1991; 111:75-78. 